\title{
Bio-efficacy different insecticides against jasmine blossom midge, Contarinia maculipennis Felt in Jasminum sambac \\ L.
}

\section{K Harini, K Elanchezhyan \& N Murugesan}

Journal of Agriculture and Ecology

ISSN: 2456-9410

Volume: 8

Journal of Agriculture and Ecology (2019) 8: 19-23 http://doi.org/10.53911/JAE.2019.8202

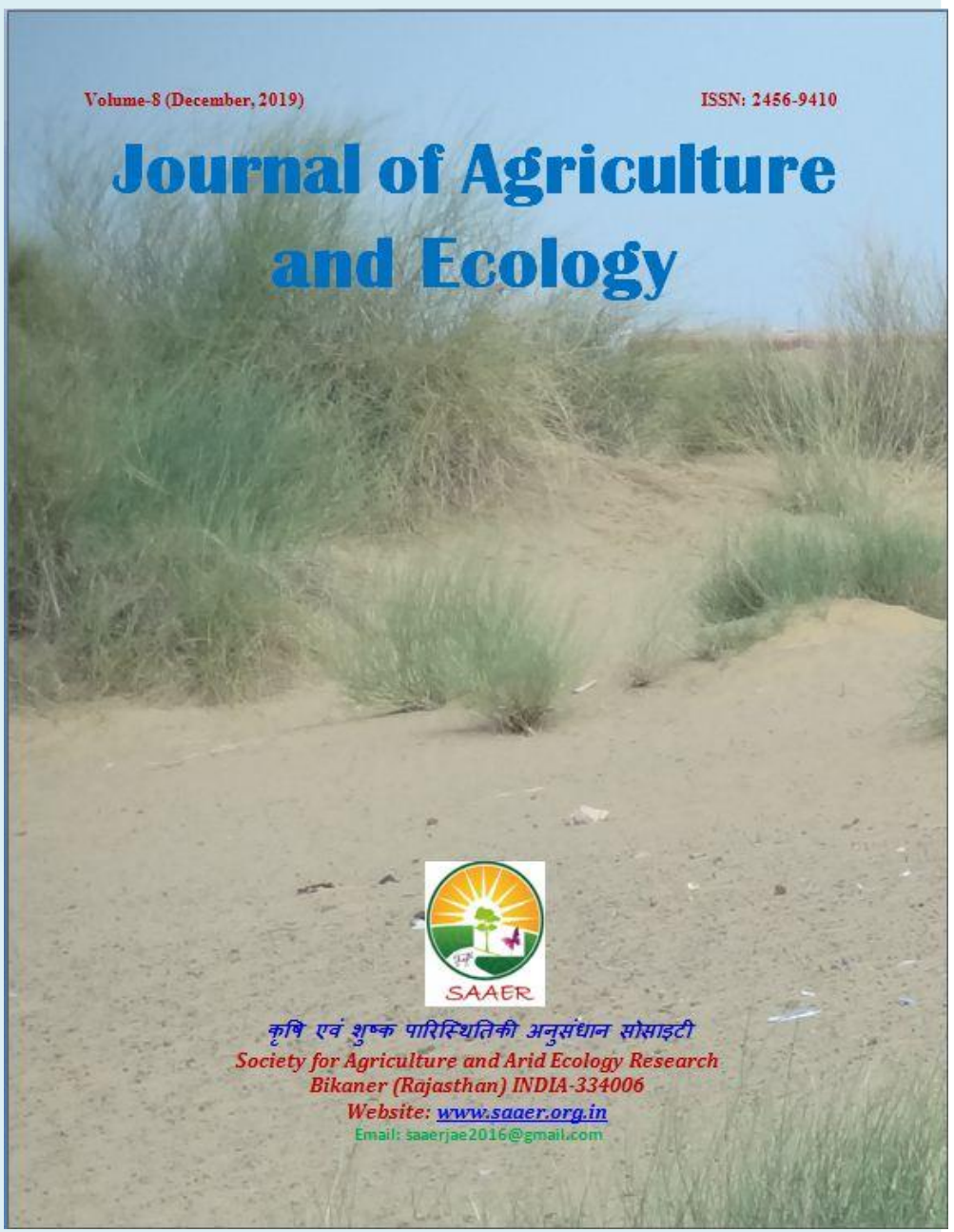




\title{
Bio-efficacy different insecticides against jasmine blossom midge, Contarinia maculipennis Felt in Jasminum sambac $\mathbf{L}$.
}

\author{
K Harini $\square$, K Elanchezhyan \& N Murugesan \\ Department of Agricultural Entomology, \\ Agricultural College and Research Institute, \\ Tamil Nadu Agricultural University, Killikulam, Vallanadu-628 252, Tamil Nadu \\ D Corresponding author: K Harini, Email: harini2594@gmail.com
}

Article Info Abstract

Article history

Received: 10 September 2018

Accepted: 15 November 2018

Available online: 5 August 2019

Key Words: Bio-

efficacy, blossom midge, insecticides, Jasminum sambac.
The bio-efficacy of five botanicals and eleven insecticides were evaluated against jasmine blossom midge, Contarinia maculipennis Felt infesting Jasminum sambac. Among the botanicals, pungam oil was the most effective treatment with least infestation followed by NSKE were able to reduce the infestation by 82.66 and 76.21 per cent, respectively. Flubendiamide $39.35 \mathrm{SC}$ was the best treatment recording the greatest reduction of 85.47 per cent followed by chlorantraniliprole $18.5 \mathrm{SC}$, respectively. The results concluded that insecticides are more effective then botanic insecticides against blossom midge.

Copyright (C2019 Harini et al., This is an open access article published under the terms of the Creative Commons Attribution License, which permits unrestricted use, distribution, and reproduction in any medium, provided the original work is properly cited.

Preferred citation: Harini K, Elanchezhyan K \& Murugesan N. 2019. Bio-efficacy different insecticides against jasmine blossom midge, Contarinia maculipennis Felt in Jasminum sambac L.. Journal of Agriculture and Ecology, 8: 19-23; http://doi.org/10.53911/JAE.2019.8202.

\section{Introduction}

Jasmine (Jasminum sambac L.) is an attractive important commercial crop in India. The importance of jasmine flower is felt in all religious, social, cultural ceremonies and other functions performed by all religious people. There are around 50 distinctive insect-pests species having a place within excess of eight orders harbouring fluctuated microhabitats of jasmine plants (Hemalatha 2009). Jasmine budworm (Hendecasis duplifascialis Hampson), blossom midge (C. maculipennis), leaf webworm (Nausinoe geometralis Guenee) and sucking insect viz., whitefly (Dialeurodes kirkaldyi Kotinsky), thrips (Isothrips orientalis Bagnall), lacewing bug (Corythauma ayyari Drake), red spider mite (Tetranychus urticae Koch) and eriophyid mite (Aceria jasmine Chanana) which are economically important insect-pests next to budworm cause economic losses to jasmine growers. At present, farmers depend mostly on conventional insecticides and acaricides for managing the jasmine insect-pests. This can lead to problems like resurgence, residue and resistance on jasmine ecosystem. Decreased efficacy of conventional pesticides as well as 
increased concerns over their use in jasmine ecosystem have emphasised on the need for identifying safer, more effective botanical, insecticide and acaricide molecules for management of jasmine insect-pests.

\section{Materials and Methods}

A field experiment was conducted in a farmer's field near Vallanad village, during the period of 2017-2018 to test the efficacy of selected botanicals and insecticides against blossom midge, Contarinia maculipennis. Randomized block design (RBD) was adopted in each treatment; three plants and three replications were maintained for observation. Three rounds of foliar sprays were given at fortnight interval using battery operated hand sprayer. C. maculipennis infestation was assessed from 10 randomly selected jasmine bushes on the basis of flowers that were discoloured to purple. Total number of flower buds and the number of flower buds showing purple discolouration due to blossom midge were recorded and the per cent damage was worked out.

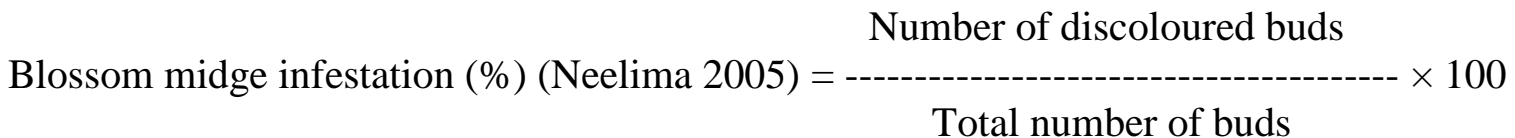

Pre-treatment observations on the incidence of blossom midge were recorded one day before spraying. Post treatment counts were recorded on $1^{\text {st }}, 3^{\text {rd }}, 7^{\text {th }}$ and $14^{\text {th }}$ day after imposing treatment. The percentage data gathered were transformed into angular values for statistical scrutiny as suggested by Gomez \& Gomez (1984).

\section{Results and Discussion}

\section{Bio-efficacy of botanicals against blossom midge, $C$. maculipennis}

The data on bio-efficacy studies with botanicals shown that the infestation of blossom midge ranged from 4.36 to 33.46 per cent (Table 1). Statistical investigation established that the significant influence of treatments, spray rounds and period of observations; interaction effect was also conspicuous. Overall mean indicated that pungam oil was the most effective treatment with least infestation $(5.53 \%)$ as well as the greatest reduction $(82.66 \%)$ in blossom midge damage. NSKE $(7.59 \%)$ was the next best treatment followed by notchi ( $V$. negundo) leaf extract $(8.24 \%)$, and wild sage ( $L$. camara) leaf extract $(9.57 \%)$; they brought out a reduction of more than 70 per cent. Sweet flag (A. calamus) treated plants recorded lesser infestation $(9.77 \%)$ than untreated plants, nevertheless inferior to former treatments. The similar trend was observed at each spray rounds as well as period of observations. The effectiveness of pungam oil @ 2.0 per cent for the control of jasmine pests revealed in the present study is confirmed by (Ponsekha \& Muthusamy 2016) with their laboratory bioassay as well as the field studies reported by (Merlin Kamala 2017) in jasmine. The efficacy of NSKE @ 5.0 per cent in managing jasmine pest brought out in the present study was also supported by (Hemalatha 2009). 
Table 1. Bio-efficacy of botanicals against blossom midge, C. maculipennis

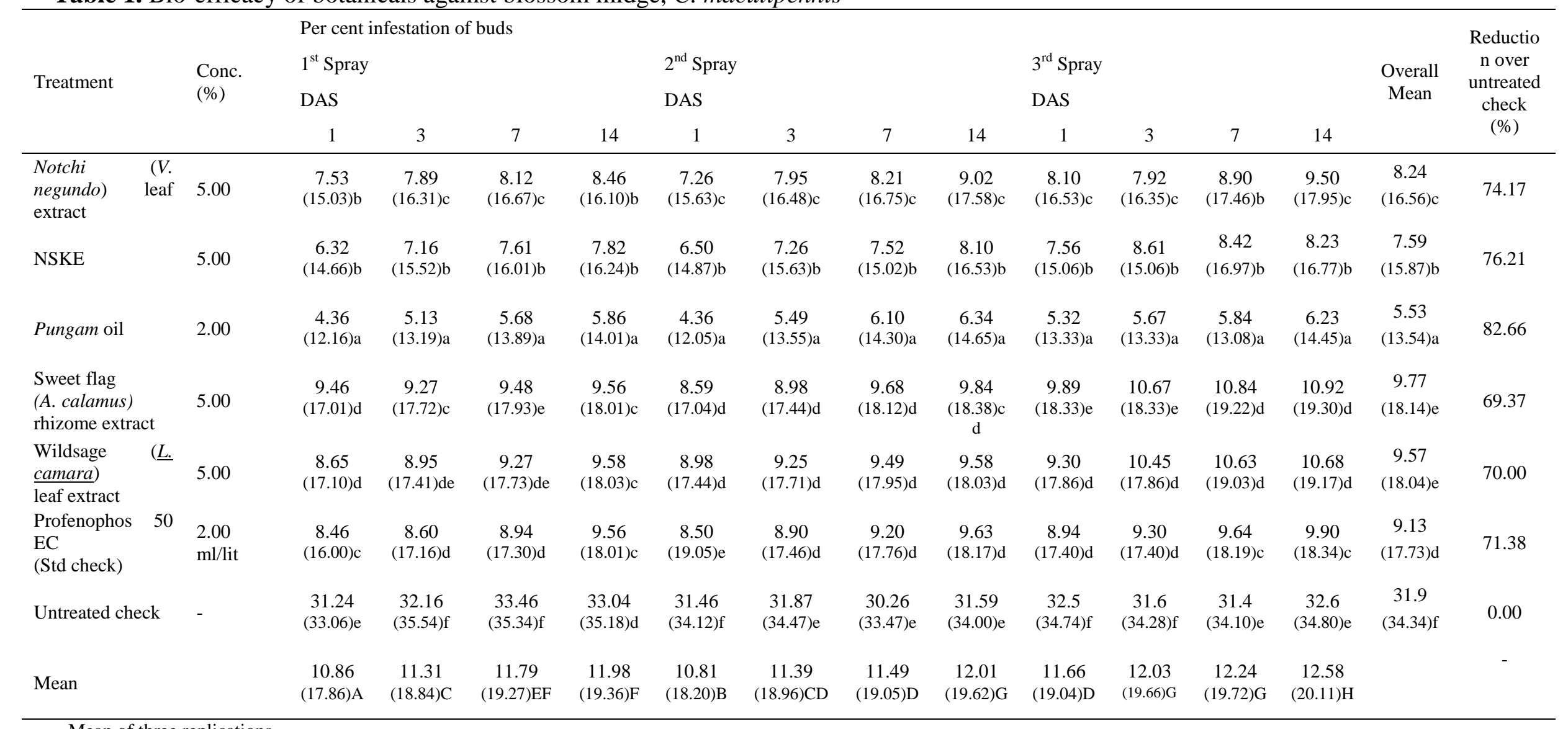

Mean of three replications.

Figures in parentheses are square root transformed values.

In a column/row, means followed by a common letter are not significantly different at 5\% level (LSD).

$\begin{array}{cccccc} & \mathrm{T} & \mathrm{S} & \mathrm{D} & \mathrm{S} \times \mathrm{D} & \mathrm{T} \times \mathrm{S} \times \mathrm{D} \\ \text { Significance } & 0.01 & 0.01 & 0.01 & 0.01 & 0.01 \\ \mathrm{CD}(\mathrm{P}=0.05) & 0.14 & 0.09 & 0.11 & 0.19 & 0.50\end{array}$


Journal of Agriculture and Ecology, 2019, Vol. 8, 19-23

http://saaer.org.in

Table 2. Bio-efficacy of insecticides against blossom midge, C. maculipennis

\begin{tabular}{|c|c|c|c|c|c|c|c|c|c|c|c|c|c|c|c|}
\hline \multirow[t]{2}{*}{ Treatment } & \multirow[t]{2}{*}{ Dose } & \multicolumn{4}{|c|}{$\begin{array}{l}\text { Per cent infestation of buds } \\
1^{\text {st }} \text { Spray } \\
\text { DAS }\end{array}$} & \multicolumn{4}{|l|}{$\begin{array}{l}2^{\text {nd }} \text { Spray } \\
\text { DAS }\end{array}$} & \multicolumn{4}{|l|}{$\begin{array}{l}3^{\text {rd }} \text { Spray } \\
\text { DAS }\end{array}$} & \multirow[t]{2}{*}{$\begin{array}{l}\text { Overall } \\
\text { Mean }\end{array}$} & \multirow{2}{*}{$\begin{array}{l}\text { Reductiol } \\
\text { over } \\
\text { untreated } \\
\text { check } \\
(\%)\end{array}$} \\
\hline & & 1 & 3 & 7 & 14 & 1 & 3 & 7 & 14 & 1 & 3 & 7 & 14 & & \\
\hline Acetamiprid $20 \mathrm{SP}$ & $0.10 \mathrm{~g} / \mathrm{lit}$ & $\begin{array}{l}6.29 \\
(14.53) \mathrm{c}\end{array}$ & $\begin{array}{l}7.09 \\
(15.44) \mathrm{c}\end{array}$ & $\begin{array}{l}7.59 \\
(15.99) \mathrm{c}\end{array}$ & $\begin{array}{l}7.81 \\
(16.23) \mathrm{d}\end{array}$ & $\begin{array}{l}6.49 \\
(14.86) \mathrm{c}\end{array}$ & $\begin{array}{l}7.26 \\
(15.63) \mathrm{e}\end{array}$ & $\begin{array}{l}7.49 \\
(15.88) \mathrm{d}\end{array}$ & $\begin{array}{l}8.06 \\
(16.50) \mathrm{e}\end{array}$ & $\begin{array}{l}7.54 \\
(15.93) \mathrm{e}\end{array}$ & $\begin{array}{l}8.60 \\
(17.15) \mathrm{ef}\end{array}$ & $\begin{array}{l}8.40 \\
(16.85) \mathrm{d}\end{array}$ & $\begin{array}{l}8.20 \\
(16.64) \mathrm{c}\end{array}$ & $\begin{array}{l}7.57 \\
(15.97) \mathrm{d}\end{array}$ & 71.29 \\
\hline $\begin{array}{l}\text { Chlorantraniliprole } 18.5 \\
\text { SC }\end{array}$ & $0.10 \mathrm{ml} /$ lit & $\begin{array}{l}4.03 \\
(11.68) \mathrm{b}\end{array}$ & $\begin{array}{l}4.56 \\
(12.33) \mathrm{a}\end{array}$ & $\begin{array}{l}4.89 \\
(12.87) \mathrm{a}\end{array}$ & $\begin{array}{l}5.13 \\
(13.09) \mathrm{a}\end{array}$ & $\begin{array}{l}4.45 \\
(12.28) \mathrm{a}\end{array}$ & $\begin{array}{l}4.97 \\
(12.99) \mathrm{a}\end{array}$ & $\begin{array}{l}5.46 \\
(13.50) \mathrm{a}\end{array}$ & $\begin{array}{l}5.87 \\
(14.02) \mathrm{b}\end{array}$ & $\begin{array}{l}3.80 \\
(11.24) \mathrm{b}\end{array}$ & $\begin{array}{l}4.60 \\
(12.48) \mathrm{a}\end{array}$ & $\begin{array}{l}5.60 \\
(13.79) \mathrm{b}\end{array}$ & $\begin{array}{l}6.00 \\
(14.28) \mathrm{b}\end{array}$ & $\begin{array}{l}4.95 \\
(12.88) \mathrm{a}\end{array}$ & 85.47 \\
\hline Dimethoate 30 EC & $2.00 \mathrm{ml} /$ lit & $\begin{array}{l}6.48 \\
(14.74) \mathrm{c}\end{array}$ & $\begin{array}{l}7.15 \\
(15.51) \mathrm{cd}\end{array}$ & $\begin{array}{l}7.68 \\
(16.19) \mathrm{c}\end{array}$ & $\begin{array}{l}7.98 \\
(16.41) \mathrm{d}\end{array}$ & $\begin{array}{l}6.49 \\
(14.86) \mathrm{c}\end{array}$ & $\begin{array}{l}7.58 \\
(15.09) \mathrm{d}\end{array}$ & $\begin{array}{l}7.89 \\
(16.31) \mathrm{e}\end{array}$ & $\begin{array}{l}8.49 \\
(16.94) f\end{array}$ & $\begin{array}{l}7.56 \\
15.06() \mathrm{d}\end{array}$ & $\begin{array}{l}8.80 \\
(17.35) \mathrm{fg}\end{array}$ & $\begin{array}{l}8.59 \\
(17.04) \mathrm{e}\end{array}$ & $\begin{array}{l}8.67 \\
(17.12) \mathrm{d}\end{array}$ & $\begin{array}{l}7.78 \\
(16.05) \mathrm{d}\end{array}$ & 75.63 \\
\hline Fipronil 5 SC & $1.50 \mathrm{ml} /$ lit & $\begin{array}{l}8.46 \\
(16.91) \mathrm{e}\end{array}$ & $\begin{array}{l}8.59 \\
(17.04) \mathrm{f}\end{array}$ & $\begin{array}{l}8.89 \\
(17.35) \mathrm{e}\end{array}$ & $\begin{array}{l}9.45 \\
(17.91) \mathrm{f}\end{array}$ & $\begin{array}{l}8.46 \\
(16.01) \mathrm{e}\end{array}$ & $\begin{array}{l}8.86 \\
(17.32) \mathrm{g}\end{array}$ & $\begin{array}{l}9.18 \\
(17.64) f\end{array}$ & $\begin{array}{l}9.53 \\
(17.98) \mathrm{gh}\end{array}$ & $\begin{array}{l}8.94 \\
(17.40) \mathrm{g}\end{array}$ & $\begin{array}{l}9.26 \\
(17.72) \mathrm{g}\end{array}$ & $\begin{array}{l}9.59 \\
(18.04) f\end{array}$ & $\begin{array}{l}9.84 \\
(18.38) \mathrm{f}\end{array}$ & $\begin{array}{l}9.09 \\
(17.48) \mathrm{h}\end{array}$ & 71.53 \\
\hline Flubendiamide $39.35 \mathrm{SC}$ & $0.75 \mathrm{ml} /$ lit & $\begin{array}{l}3.59 \\
(10.92) \mathrm{a}\end{array}$ & $\begin{array}{l}4.26 \\
(11.92) \mathrm{a}\end{array}$ & $\begin{array}{l}4.59 \\
(12.47) \mathrm{a}\end{array}$ & $\begin{array}{l}4.87 \\
(12.75) \mathrm{a}\end{array}$ & $\begin{array}{l}4.25 \\
(17.90) \mathrm{g}\end{array}$ & $\begin{array}{l}4.86 \\
(12.74) \mathrm{a}\end{array}$ & $\begin{array}{l}5.31 \\
(13.33) \mathrm{s}\end{array}$ & $\begin{array}{l}5.49 \\
(13 . a 55)\end{array}$ & $\begin{array}{l}3.46 \\
(10.72) \mathrm{a}\end{array}$ & $\begin{array}{l}4.40 \\
(12.11) \mathrm{a}\end{array}$ & $\begin{array}{l}5.23 \\
(13.22) \mathrm{a}\end{array}$ & $\begin{array}{l}5.40 \\
(13.43) \mathrm{a}\end{array}$ & $\begin{array}{l}4.64 \\
(12.92) \mathrm{a}\end{array}$ & 84.50 \\
\hline Imidacloprid 17.8 SL & $0.30 \mathrm{ml} /$ lit & $\begin{array}{l}7.26 \\
(15.63) \mathrm{d}\end{array}$ & $\begin{array}{l}7.49 \\
(15.88) \mathrm{de}\end{array}$ & $\begin{array}{l}7.89 \\
(16.32) \mathrm{cd}\end{array}$ & $\begin{array}{l}8.42 \\
(16.87) \mathrm{e}\end{array}$ & $\begin{array}{l}7.08 \\
(15.43) \mathrm{d}\end{array}$ & $\begin{array}{l}7.69 \\
(16.10) f\end{array}$ & $\begin{array}{l}7.84 \\
(16.36) \mathrm{e}\end{array}$ & $\begin{array}{l}8.67 \\
(17 . f 12)\end{array}$ & $\begin{array}{l}7.69 \\
\text { (16.10)ef }\end{array}$ & $\begin{array}{l}8.30 \\
(16.74) \text { ef }\end{array}$ & $\begin{array}{l}8.59 \\
(17.04) \mathrm{e}\end{array}$ & $\begin{array}{l}9.12 \\
(17.68) \mathrm{e}\end{array}$ & $\begin{array}{l}8.00 \\
(16.44) \mathrm{e}\end{array}$ & 74.94 \\
\hline Indoxacarb $14.5 \mathrm{SC}$ & $0.35 \mathrm{ml} /$ lit & $\begin{array}{l}9.02 \\
(17.57) \mathrm{f}\end{array}$ & $\begin{array}{l}9.25 \\
(17.71) \mathrm{g}\end{array}$ & $\begin{array}{l}9.46 \\
(17.92) f\end{array}$ & $\begin{array}{l}9.80 \\
(18.24) \mathrm{f}\end{array}$ & $\begin{array}{l}8.80 \\
(17.36) f\end{array}$ & $\begin{array}{l}9.26 \\
(17.72) \mathrm{g}\end{array}$ & $\begin{array}{l}9.48 \\
(17.92) f\end{array}$ & $\begin{array}{l}9.89 \\
(18 . h 33)\end{array}$ & $\begin{array}{l}9.56 \\
(18.01) \mathrm{h}\end{array}$ & $\begin{array}{l}10.26 \\
(18.78) \mathrm{h}\end{array}$ & $\begin{array}{l}10.49 \\
(18.90) \mathrm{g}\end{array}$ & $\begin{array}{l}10.89 \\
(19.37) \mathrm{g}\end{array}$ & $\begin{array}{l}9.68 \\
(18.15) \mathrm{j}\end{array}$ & 69.68 \\
\hline Novaluron $10 \mathrm{EC}$ & $1.00 \mathrm{ml} /$ lit & $\begin{array}{l}8.64 \\
(17.19) \text { ef }\end{array}$ & $\begin{array}{l}8.95 \\
(17.41) f g\end{array}$ & $\begin{array}{l}9.26 \\
(17.71) \text { ef }\end{array}$ & $\begin{array}{l}9.54 \\
(17.90) f\end{array}$ & $\begin{array}{l}8.97 \\
(17.43) \mathrm{f}\end{array}$ & $\begin{array}{l}9.23 \\
(17.68) \mathrm{g}\end{array}$ & $\begin{array}{l}9.48 \\
(17.93) f\end{array}$ & $\begin{array}{l}9.48 \\
(17.9 \mathrm{gh} 3)\end{array}$ & $\begin{array}{l}9.26 \\
(17.72) \mathrm{gh}\end{array}$ & $\begin{array}{l}10.43 \\
(18.85) \mathrm{gh}\end{array}$ & $\begin{array}{l}10.59 \\
(18.00) f\end{array}$ & $\begin{array}{l}10.65 \\
(19.05) \mathrm{g}\end{array}$ & $\begin{array}{l}9.54 \\
(17.90) \mathrm{i}\end{array}$ & 70.12 \\
\hline Spinosad 45 SC & $0.32 \mathrm{ml} /$ lit & $\begin{array}{l}7.46 \\
(15.85) d\end{array}$ & $\begin{array}{l}7.89 \\
(16.32) \mathrm{e}\end{array}$ & $\begin{array}{l}8.04 \\
(16.57) d\end{array}$ & $\begin{array}{l}8.46 \\
(16.91) \mathrm{e}\end{array}$ & $\begin{array}{l}7.26 \\
(15.63) \mathrm{de}\end{array}$ & $\begin{array}{l}7.86 \\
(16.28) f\end{array}$ & $\begin{array}{l}8.16 \\
(16.60) \mathrm{e}\end{array}$ & $\begin{array}{l}9.02 \mathrm{~g} \\
(17.58)\end{array}$ & $\begin{array}{l}8.06 \\
(16.50) \mathrm{f}\end{array}$ & $\begin{array}{l}7.90 \\
(16.33) \mathrm{f}\end{array}$ & $\begin{array}{l}8.90 \\
(17.45) \mathrm{e}\end{array}$ & $\begin{array}{l}9.50 \\
(17.06) \mathrm{cd}\end{array}$ & $\begin{array}{l}8.21 \\
(16.59) f\end{array}$ & 74.29 \\
\hline Thiacloprid 21.7 SC & $0.60 \mathrm{ml} /$ lit & $\begin{array}{l}6.23 \\
(14.44) \mathrm{c}\end{array}$ & $\begin{array}{l}5.32 \\
(13.33) \mathrm{b}\end{array}$ & $\begin{array}{l}6.13 \\
(14.33) \mathrm{c}\end{array}$ & $\begin{array}{l}6.49 \\
(14.86) \mathrm{c}\end{array}$ & $\begin{array}{l}5.64 \\
(13.74) b\end{array}$ & $\begin{array}{l}6.28 \\
(14.51) \mathrm{c}\end{array}$ & $\begin{array}{l}6.94 \\
(15.37) c\end{array}$ & $\begin{array}{l}7.05 \mathrm{~d} \\
(15.40)\end{array}$ & $\begin{array}{l}6.46 \\
(14.72) \mathrm{d}\end{array}$ & $\begin{array}{l}6.84 \\
(15.26) \mathrm{d}\end{array}$ & $\begin{array}{l}7.14 \\
(15.50) \mathrm{c}\end{array}$ & $\begin{array}{l}7.29 \\
(16.76) \mathrm{cd}\end{array}$ & $\begin{array}{l}6.48 \\
(14.85) c\end{array}$ & 79.71 \\
\hline Thiamethoxam $25 \mathrm{WG}$ & $0.40 \mathrm{~g} / \mathrm{lit}$ & $\begin{array}{l}4.35 \\
(12.03) \mathrm{b}\end{array}$ & $\begin{array}{l}5.12 \\
(13.18) \mathrm{b}\end{array}$ & $\begin{array}{l}5.64 \\
\quad(13.74) \mathrm{b}\end{array}$ & $\begin{array}{l}5.84 \\
(13.98) \mathrm{b}\end{array}$ & $\begin{array}{l}4.35 \\
(12.03) \mathrm{a}\end{array}$ & $\begin{array}{l}5.43 \\
(13.57) \mathrm{b}\end{array}$ & $\begin{array}{l}6.01 \\
(14.19) \mathrm{b}\end{array}$ & $\begin{array}{l}6.23 \mathrm{c} \\
(14.55)\end{array}$ & $\begin{array}{l}5.32 \\
(13.33) \mathrm{c}\end{array}$ & $\begin{array}{l}5.67 \\
(13.88) \mathrm{c}\end{array}$ & $\begin{array}{l}5.89 \\
(14.04) \mathrm{b}\end{array}$ & $\begin{array}{l}6.10 \\
(14.30) b\end{array}$ & $\begin{array}{l}5.50 \\
(13.57) \mathrm{b}\end{array}$ & 82.77 \\
\hline Untreated check & - & $\begin{array}{l}31.24 \\
(33.98) \mathrm{g}\end{array}$ & $\begin{array}{l}32.16 \\
(34.55) \mathrm{h}\end{array}$ & $\begin{array}{l}33.46 \\
(35.34) \mathrm{g}\end{array}$ & $\begin{array}{l}33.04 \\
(35.19) \mathrm{g}\end{array}$ & $\begin{array}{l}31.46 \\
(34.12) \mathrm{h}\end{array}$ & $\begin{array}{l}31.87 \\
(34.57) \mathrm{h}\end{array}$ & $\begin{array}{l}30.26 \\
(33.37) \mathrm{g}\end{array}$ & $\begin{array}{l}31.59 \\
(34.20) \mathrm{i}\end{array}$ & $\begin{array}{l}32.48 \\
(34.75) \mathrm{i}\end{array}$ & $\begin{array}{l}31.56 \\
(34.28) \mathrm{i}\end{array}$ & $\begin{array}{l}31.44 \\
(34.12) \mathrm{h}\end{array}$ & $\begin{array}{l}32.58 \\
(34.80) \mathrm{h}\end{array}$ & $\begin{array}{l}31.93 \\
(34.44) \mathrm{k}\end{array}$ & 0.00 \\
\hline Mean & & $\begin{array}{l}8.59 \\
(16.29) \mathrm{A}\end{array}$ & $\begin{array}{l}8.99 \\
(16.72) \mathrm{B}\end{array}$ & $\begin{array}{l}9.46 \\
(17.23) \mathrm{D}\end{array}$ & $\begin{array}{l}9.74 \\
(17.53) \mathrm{F}\end{array}$ & $\begin{array}{l}8.64 \\
(16.80) \mathrm{B}\end{array}$ & $\begin{array}{l}9.26 \\
(17.02) \mathrm{C}\end{array}$ & $\begin{array}{l}9.46 \\
(17.37) \mathrm{E}\end{array}$ & $\begin{array}{l}9.95 \\
(17.84) \mathrm{G}\end{array}$ & $\begin{array}{l}9.18 \\
(16.79) \mathrm{B}\end{array}$ & $\begin{array}{l}9.72 \\
(17.58) \mathrm{F}\end{array}$ & $\begin{array}{l}10.04 \\
(17.83) \mathrm{G}\end{array}$ & $\begin{array}{l}10.35 \\
(18.24) \mathrm{H}\end{array}$ & & - \\
\hline
\end{tabular}

Mean of three replications. Figures in parentheses are square root transformed values.

In a column/row, means followed by a common letter are not significantly different at $5 \%$ level (LSD).

$\begin{array}{lccccc} & \mathrm{T} & \mathrm{S} & \mathrm{D} & \mathrm{S} \times \mathrm{D} & \mathrm{T} \times \mathrm{S} \times \mathrm{D} \\ \text { Significance } & 0.01 & 0.01 & 0.01 & 0.01 & 0.01 \\ \mathrm{CD}(\mathrm{P}=0.05) & 0.12 & 0.06 & 0.07 & 0.12 & 0.42\end{array}$


Bio-efficacy of insecticides against blossom midge, $C$. maculipennis

The data from the field trial in the bioefficacy of insecticides against blossom midge shown the percentage infestation on the bud of jasmine ranged from 3.59 to 33.46 per cent. The statistical analysis of the data brought out the marked influence of treatments, spray rounds and period of observations; interaction effect was also obvious (Table 2). Considering the spray rounds, as well as period of observations together flubendiamide $39.35 \mathrm{SC}$ $(4.64 \%)$ as well as chlorantraniliprole 18.5 SC $(4.95 \%)$ were found to be the most effective treatments recording the minimum infestation; they could assert a reduction of infestation by more than 84 per cent compared to the untreated plants. Thiamethoxam $25 \mathrm{WG}$ $(5.50 \%)$ was the next best treatment recording 82.77 per cent reduction over untreated check. A reduction in infestation by less than 70 per cent reduction in bud damage was observed on thiacloprid 21.7 SC, acetamiprid 20 SP, dimethoate $30 \mathrm{EC}$, imidacloprid $17.8 \mathrm{SL}$, spinosad $45 \mathrm{SC}$, fipronil $5 \mathrm{SC}$ and novaluron 10 EC treated plants; they recorded an infestation of $6.48,7.57,7.78,8.00,8.21,9.09$ and 9.54 per cent respectively. Indoxacarb 14.5 SC was the least effective treatment with 69.68 per cent, reduction in bud infestation. Chlorantraniliprole18.5 SC @ $0.10 \mathrm{ml} / \mathrm{lit}$ and flubendiamide39.35 SC @ 0.75 ml/lit belongs to the main group of ryanodine receptor modulators and chemical sub group of diamides (IRAC 2009). They inhibit the nerve and muscle action in insects. These two insecticides were used against broad spectrum of lepidopterous insects. These molecules affect intercellular $\mathrm{Ca}^{2+}$ channels (Omkar Gavkare et al. 2013; Singh et al. 2017). Merlin Kamala (2017) reported that thiacloprid 240 SC @ $0.6 \mathrm{ml} /$ lit proved its superiority in managing blossom midge.

\section{References}

Gomez KA, and Gomez AA. 1984. Statistical Procedures for Agricultural Research: John Wiley \& Sons, New York. 680p.

Hemalatha G. 2009. Biorational management of key pests of Jasmine (Jasminum sambac). College of Horticulture, Vellanikkara.

IRAC. 2009. IRAC Mode of Action Classification Scheme. Insecticide Resistance Action Committee, New Delhi pp: 1-26.

Merlin Kamala I. 2017. Studies on diversity, bioecology and integrated management of major pests of jasmine (Jasminum sambac L.). (Ph. D.Thesis), Tamil Nadu Agricultural University. AgriculturalCollege and Research Institute, Madurai. 410p.

Neelima Y. 2005. Bioecology and management of jasmine pests. Acharya N G Ranga Agricultural University, Rajendranagar, Hyderabad.

Omkar Gavkare, Meena UP, Kulkarni AV \& Gupta S. 2013. New Group of Insecticides. Popular Kheti, 1(3): 185189.

Ponsekha CP \& Muthusamy M. 2016. Effect of neem oil, pungam oil and monocrotophos against the larvae of jasmine budworm Hendecasis duplifacialis (Pyraustidae: Lepidoptera). International Journal of Advanced Life Sciences, 9(2): 185-189.

Singh H, Deshwal HL, Haldhar SM and Singh V. 2017. Bio-efficacy of some insecticides and botanicals against mustard aphid, Lipaphis erysimi Kalt. (Hemiptera: Aphididae) on Indian mustard. Journal of Experimental Zoology, 20 (1): 259-253. 